\title{
OBTENÇÃO E SEPARAÇÃO DE COMPOSTOS BIOATIVOS DE Schinus terebinthifolius raddi EM MEIO SUPERCRÍTICO.
}

\author{
M.A. LIVIA ${ }^{1}$, A.W.ZIBETTI ${ }^{1}$, A. MARANGONI ${ }^{1}$ e A.BOLZAN ${ }^{1}$ \\ ${ }^{1}$ Universidade Federal de Santa Catarina, Departamento de Engenharia Química e Engenharia de \\ Alimentos \\ E-mail para contato: mayarau0@gmail.com
}

\begin{abstract}
RESUMO - Pimenta rosa (Schinus terebenthifolius Raddi), distribuída amplamente no território brasileiro, possui várias ações terapêuticas, tais como: cicatrizante, antisséptica, antifúngica e anti-inflamatória. Estudos recentes mostraram que seu óleo essencial possui também potencial citotóxico em linhagens de células cancerosas. Visando a aplicação de compostos bioativos desta planta na área biomédica, o objetivo deste trabalho foi o desenvolvimento de um protocolo para a obtenção e separação destes compostos utilizando fluido supercrítico. Para isto, diferentes condições operacionais de extração foram avaliadas. Os resultados obtidos apresentaram a existência de duas frações com diferente composição química sendo a condição de 80 bar de pressão e $35{ }^{\circ} \mathrm{C}$ a que mostrou uma melhor separação dos compostos. A primeira fração apresentou os seguintes compostos majoritários: alfa-felandreno, delta-3-carene e limoneno. Já a segunda apresentou: beta-Elemen-7 alfa-ol, Elemol e beta-elemene.
\end{abstract}

\section{INTRODUÇÃO}

$\mathrm{Na}$ indústria farmacêutica também a descoberta de novos fármacos, cada vez mais potentes e menos tóxicos é constante. Neste sentido, o reino vegetal detém as maiores fontes de substâncias ativas que podem ser usadas na terapêutica devido à grande diversidade estrutural de metabólitos produzida, sendo a fonte mais antiga de medicamentos para o homem (SAMUELSSON, 1999).

Os métodos de extração para a obtenção e separação de compostos bioativos a partir de material vegetal são diversos. Entre os métodos tradicionais podem ser citados a hidrodestilação e extração com arraste a vapor. As técnicas tradicionais apresentam desvantagens, como o emprego de alta temperatura, o que pode incorrer na degradação de compostos termo-lábeis e formação de outros compostos indesejados. Outro método comumente utilizado é a extração com solvente orgânico, porém esse processo demanda uma etapa posterior de separação, o que pode elevar os custos de produção, além da possibilidade de contaminação ambiental e da provável presença de resíduos desses solventes no produto final, restringindo a sua aplicação (MUKHOPADHYAY, 2000).

Nesse contexto, tem-se a Schinus terebinthifolius raddi, comumente conhecida como pimenta rosa, pertencente à família Anacardiaceae e amplamente distribuída ao longo da costa do território brasileiro (LORENZI, 1998; LENZI, 2004). Esta planta apresenta-se como uma fonte prospera, 


\section{9 a 22 de outubro de 2014 \\ Florianópolis/SC}

especialmente quando se considera seu uso popular abundante. Diversas partes desta são utilizadas pelas diferentes ações terapêuticas que possuem, como a cicatrizante, antisséptica, antifúngica e antiinflamatória, (VELAZQUEZ, et al., 2003; SCHMOURLO, et al., 2005; DE LIMA, et al., 2006).Além destas, recentemente o óleo essencial dos frutos extraído por hidrodestilação apresentou ação antitumoral em linhagens de células cancerosas de seio (BENDAOUD et al., 2010).

$\mathrm{Na}$ composição química dos frutos hidrodestilados de S. terebinthifolius destacam-se os seguintes compostos majoritários: $\alpha$-pineno; $\alpha$-felandreno; delta-3-careno; $\beta$-felandreno e germacrene-D. Porém, estudos realizados por Barbosa, etal. (2007) confirmam que existe uma elevada variabilidade na composição química dos óleos essenciais dos frutos desta planta, sendo relevante para uma investigação padronizar o local e tempo de colheita.

No processo de extração com dióxido de carbono em estado supercrítico isso já é, em parte, possível, pois ao modificar a densidade do solvente manipula-se a seletividade do fluido supercrítico e, com isso, a solubilidade dos compostos (BRUNNER, 1994; REVERCHON, et al., 2006). Para isto, atualmente utilizam-se separadores acoplados ao extrator e o processo de fracionamento se realiza de maneira contínua durante o processo de extração (GAÑÁN; BRIGNOLE, 2013), no entanto, o controle do processo de separação (fracionamento) é uma tarefa complexa que envolve o equilíbrio de fases ( ZIBETTI et al., 2013).

Assim, este trabalho mostra que com a utilização da tecnologia supercrítica logrou-se obter duas frações com diferente composição química do extrato de $S$. terebinthifolius sem precisar de separadores acoplados ao extrator. Para isto, diferentes condições operacionais de extração foram avaliadas e a separação do extrato foi realizada de maneira simultânea.

\section{MATERIAIS E METODOS}

Os experimentos foram realizados no Laboratório de Controle de Processos do Departamento de Engenharia Química e Engenharia de Alimentos da Universidade Federal de Santa Catarina (LCP/ENQ/UFSC).

As sementes de S. terebinthifolius R., utilizadas neste trabalho foram adquiridas da empresa Brasfoods Exportação e Importação Ltda. Todos os testes foram realizados com sementes provenientes do mesmo lote. Imediatamente após sua aquisição o material vegetal foi triturado e a casca foi retirada mediante esforço mecânico em um peneirador (Mesh Tyler 8 [2,362 mm])

.Logo após este processo foi novamente particulado e peneirado (Mesh Tyler 14 [1,168], separado, embalado hermeticamente a vácuo em saco de $500 \mathrm{~g}$, e mantido sob refrigeração (Brastemp, Modelo Frostfree, Brasil) sob temperaturas abaixo de $\left(4^{\circ} \mathrm{C}\right)$.

\subsection{Extração com dióxido de carbono em estado supercrítico}

O processo de extração com dióxido de carbono em estado supercrítico foi conduzido no equipamento HPLC-SC da Jasco Inc (Figura 1), com opção de extração e cromatografia supercrítica. 
Utilizou-se como solvente o dióxido de carbono com 99,95\% de pureza. O tempo total de extração foi 90 minutos, com em regime continuo. A vazão do $\mathrm{CO}_{2}$ supercrítico foi mantida em $3,0 \mathrm{~mL} / \mathrm{min}$ para todas as condições experimentais testadas.
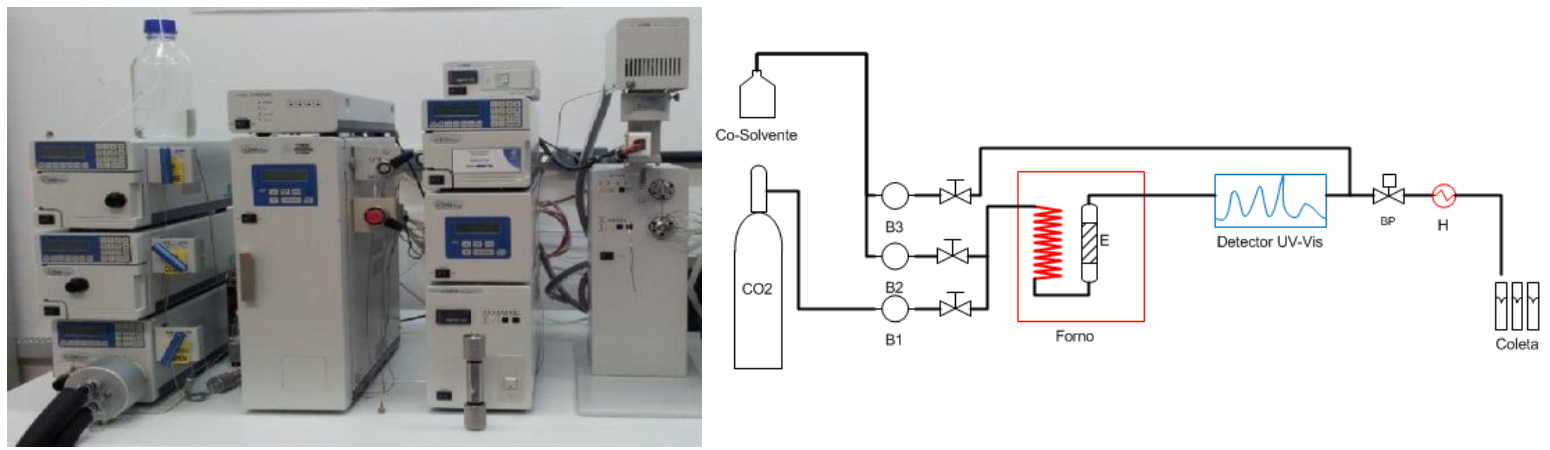

Figura 1 - Unidade de extração do laboratório de Controle de Processos: HPLC SC. B- bomba HPLC, E- extrator, BP- Válvula Backpressure, $\mathrm{H}$-Aquecimento

Como se observa na Figura 1, o HPLC-SC é composto por três bombas: bomba de $\mathrm{CO}_{2}$ (B1), bomba de cossolvente (B2) e bomba de eluente (B3); forno de aquecimento, trocador de calor, transdutores de pressão, detector UV-vis em linha, válvula back-pressure automatizada (BP), sistema de aquecimento $(\mathrm{H})$ controlado para amenizar o efeito de resfriamento provocado pela expansão do $\mathrm{CO}_{2}$ (Efeito Joule-Thompson). Utilizou-se o vaso de extração (E) com volume interno de $0,010 \mathrm{~L}$ (1,0 x 10-6 m3), com filtro de $10 \mu \mathrm{m}$ na saída do extrator, para evitar a contaminação com particulados sólidos na linha. $\mathrm{O}$ equipamento possui uma interface de comunicação LC-net II (Jasco Inc.) com o computador onde por meio do software ChromNAV podem ser controladas as variáveis operacionais (temperatura, vazão de solvente, cossolvente e eluente além da pressão mediane a back-pressure automatizada).

Os experimentos foram realizados utilizando aproximadamente 8,0 gramas de semente. Para determinação do rendimento global de extração utilizou-se um planejamento fatorial completo com cinco níveis (planejamento em estrela) conforme Tabela 1. Foram conduzidos 9 experimentos para estimar os coeficientes do modelo empírico e mais 1 experimento para determinar o erro experimental. Todos os experimentos foram realizados em triplicata.

Tabela 1: Matriz do planejamento em estrela.

\begin{tabular}{|l|c|c|c|c|c|}
\hline Fatores & $-\alpha$ & -1 & 0 & 1 & $+\alpha$ \\
\hline Temperatura & 31 & 35 & 45 & 55 & 60 \\
\hline Pressão & 80 & 100 & 150 & 200 & 220 \\
\hline
\end{tabular}


Os frascos com os extratos obtidos foram pesados em balança analítica e armazenados em freezer a $-18{ }^{\circ} \mathrm{C}$. O rendimento global $\left(X_{\mathrm{o}}\right)$ foi calculado pela da razão entre a massa de extrato $(\mathrm{m}$ extrato) obtida e a massa de $S$. terebinthifolius ( $m_{\text {semente }}$ ) utilizada para formar o leito, conforme a equação (1):

$$
X_{\mathrm{o}}=\operatorname{extrato}(\mathrm{g}) / \operatorname{semente}(\mathrm{g}) \ldots \ldots \ldots
$$

\subsection{Caracterização química dos extratos obtidos de S. terebinthifolius.}

Para a caracterização química dos extratos foram conduzidas análises Cromatografia Gasosa acoplada à Espectrômetro de Massas (GC-MS) nos laboratórios do Instituto de Pesquisas Tecnológicas de Blumenau (IPTB - FURB - Blumenau-SC). Posteriormente no laboratório de Controle de processos realizou-se análise de cromatografia gasosa em detector de ionização de chama (GC-FID).

As análises cromatográficas com espectro de massa foram realizadas com um GC-MS (Shimadzu GCMS-GC2010-QP2010 plus + AOC 5000) equipado com uma coluna RTX 5 (30m de comprimento $\mathrm{x} 0,25 \mathrm{~mm}$ de diâmetro $\mathrm{x} 0,25 \mu \mathrm{m}$ de espessura do filme) $\mathrm{O}$ gás de arraste utilizado foi hélio (He) a fluxo constante de $1 \mathrm{ml} / \mathrm{min}$. A temperatura do injetor manteve-se em $250^{\circ} \mathrm{C}$ e a temperatura do forno foi mantida a $60^{\circ} \mathrm{C}$ por um minuto e, em seguida, aumentada até $240^{\circ} \mathrm{C}$ a uma taxa de $3^{\circ} \mathrm{C} / \mathrm{min}$. No espectrômetro de massas (MS) foram utilizadas as seguintes temperaturas: temperatura de iontrap $\left(250^{\circ} \mathrm{C}\right)$, temperatura de interface $\left(280^{\circ} \mathrm{C}\right)$. O software de gerenciamento utilizado foi o GCMS Solutions da Shimadzu e as bibliotecas de compostos utilizadas para identificação foram a NIST08s e a Willey.

Análises de cromatografia gasosa em detector de ionização de chama (GC-FID) também foram realizadas. No cromatógrafo a gás, a coluna e os patamares de temperatura utilizados foram os mesmos usados na cromatografia gasosa acoplada à espectrometria de massas (GC-MS). O detector de ionização de chama foi mantido a $280^{\circ} \mathrm{C}$ e o gás de arraste, $\mathrm{He}$, a fluxo constante de $1,2 \mathrm{ml} / \mathrm{min}$. Foi utilizado o mesmo software de gerenciamento.

\subsection{Separação da composição química extratos utilizando fluido supercrítico}

Para separação química dos extratos supercríticos de S.terebinthifolius foi determinada a metodologia durante a construção das curvas de extração com ajuda da leitura do gráfico UV do HPLC-SC. Este detector possui a característica de fazer a varredura pontual durante o processo enquanto está sendo extraído o material vegetal. A leitura foi realizada em $254 \mathrm{~nm}$. Esta observação determinou a coleta de duas frações durante o processo de extração supercrítica.

Segundo mostra a Figura 2, houve uma mudança na coloração dos extratos ao longo do tempo da extração. Assim, a fração 1 apresentou uma coloração amarelo clara e corresponde aos primeiros minutos do processo. Já a fração 2 apresenta uma coloração amarelo escura correspondente aos minutos restantes. 


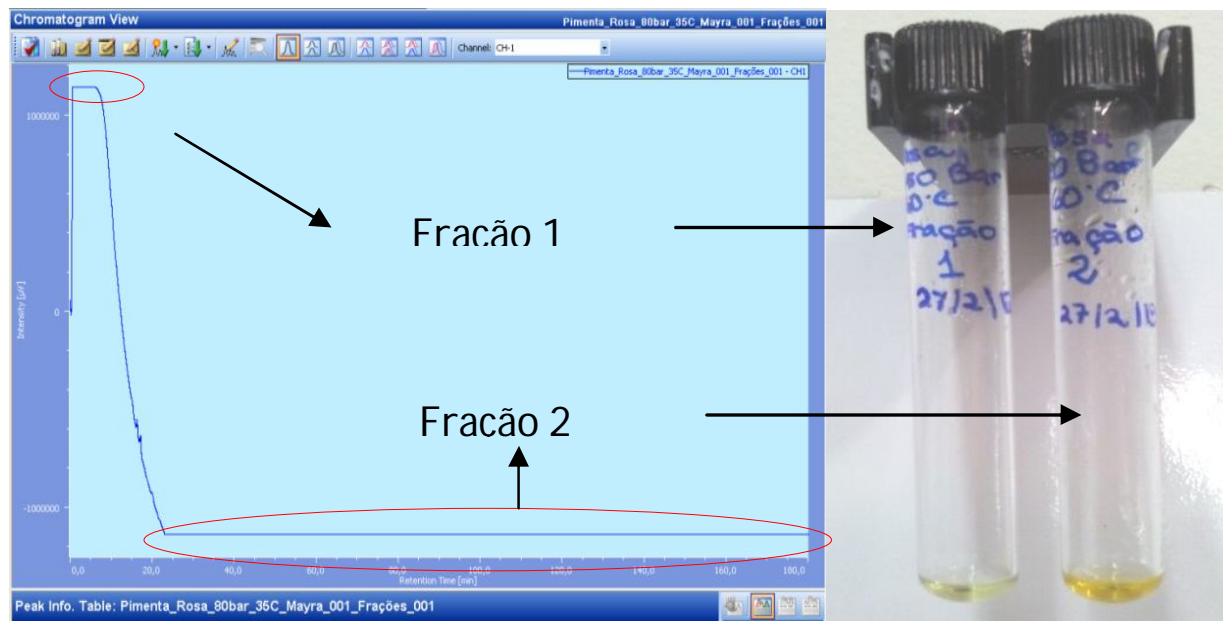

Figura 2 - Leitura do gráfico UV durante a extração supercrítica de $S$. terebenthifolius apresentando duas regiões (fração 1 e fração 2) com seus extratos correspondentes.

Desta maneira se padronizou o momento da coleta durante o processo obtendo-se duas frações para cada condição experimental como descrito na Tabela 2.

Tabela 2- Tempo de coleta da fração 1 e a fração 2

\begin{tabular}{|l|c|}
\hline Fração & Tempo de coleta \\
\hline 1 & Do inicio até o minuto 4. \\
\hline 2 & Do minuto 5 até o minuto 180. \\
\hline
\end{tabular}

Para análise da composição química foram selecionadas 4 amostras segundo a Tabela 3, tendo em consideração a maior e menor pressão operacional do planejamento experimental $\left(80\right.$ bar e $35^{\circ} \mathrm{C}$ e 220 bar e $45^{\circ} \mathrm{C}$ ). As identificações dos extratos analisados estão descritos na Tabela 3.

Tabela 3- Identificação dos extratos analisados por GC-MS e GC-FID

\begin{tabular}{|c|c|}
\hline Condição operacional & Fração \\
\hline 80 bar e $35^{\circ} \mathrm{C}$ & 1 \\
\hline 80 bar e $35^{\circ} \mathrm{C}$ & 2 \\
\hline $45^{\circ} \mathrm{Ce} 220 \mathrm{bar}$ & 1 \\
\hline $45^{\circ} \mathrm{C}$ e $220 \mathrm{bar}$ & 2 \\
\hline
\end{tabular}

\section{RESULTADOS E DISCUSSÃO}

\subsection{Rendimento Global da Extração Supercrítica $\left(X_{0}\right)$}

Os resultados de rendimento global de extração $\left(X_{\circ}\right)$ de $S$. terebinthifolius $c o m \mathrm{CO}_{2}$ supercrítico puro são apresentados na Tabela 4 , com os valores de temperatura $(\mathrm{T})$, pressão $(\mathrm{P})$ e densidade de 
solvente $\left(\rho \mathrm{CO}_{2}\right)$ correspondente. $\mathrm{O}$ rendimento global da extração é definido como a quantidade de extrato presente na matriz sólida possível de ser extraído pelo solvente nas condições estabelecidas de temperatura e pressão para um tempo de processo pré-determinado (MARTÍNEZ et al . 2005).

Os resultados que se mostram no planejamento experimental mostraram que os melhores rendimentos foram obtidos na medida em que as densidades foram mais elevadas. Assim, se obteve o melhor rendimento na condição de 150 bar e $31^{\circ} \mathrm{C}$ com 10,38\%. Já na condição de 80 bar e $45{ }^{\circ} \mathrm{C}$ se obteve o menor rendimento. De acordo com McHugh e Krukonis (1994), o poder solvente de um fluido sub ou supercrítico está relacionado com sua densidade e, desta forma, espera-se que maiores variações de densidade entre duas condições experimentais proporcionem maiores variações no rendimento final de extrato.

Tabela 4 - Rendimento obtido a partir do planejamento em estrela.

\begin{tabular}{l|lllll}
\hline Ensaio & Temperatura $\left({ }^{\circ} \mathrm{C}\right)$ & Pressão (bar) & $\rho \mathrm{CO}_{2}\left(\mathrm{Kg} / \mathrm{m}^{3}\right)$ & $X_{0}$ & Desvio padrão $( \pm)$ \\
\hline 1 & 35 & 100 & 712,8 & 5,50 & 0,07 \\
2 & 35 & 200 & 865,7 & 9,03 & 0,04 \\
3 & 55 & 100 & 325,07 & 1,98 & 0,04 \\
4 & 55 & 200 & 754,6 & 9,99 & 0,06 \\
\hline 5 & 31 & 150 & 840,8 & 10,38 & 0,05 \\
\hline 6 & 60 & 150 & 604,09 & 7,83 & 0,06 \\
\hline 7 & 45 & 80 & 241,05 & 1,54 & 0,04 \\
\hline 8 & 45 & 220 & 832,36 & 8,17 & 0,06 \\
9 & 45 & 150 & 741,97 & 8,59 & 0,04 \\
10 & 45 & 150 & 741,97 & 9,24 & 0,07 \\
\hline
\end{tabular}

\subsection{Composição química dos extratos separados.}

Foram identificados 16 compostos como se mostra a seguir na Tabela 5:

Tabela 5 - Composição química (\% areaGC-MS) dos extratos fracionados de S. terebinthifolius.

\begin{tabular}{|c|c|c|c|c|c|c|}
\hline$N^{\circ}$ & $R T$ & Composto & $\begin{array}{l}80 \text { bar } \\
35^{\circ} \mathrm{C} \\
\text { Fração1 }\end{array}$ & $\begin{array}{l}80 \mathrm{bar} \\
35^{\circ} \mathrm{C} \\
\text { Fração } 2\end{array}$ & $\begin{array}{l}220 \text { bar } \\
45^{\circ} \mathrm{C} \\
\text { Fração } 1\end{array}$ & $\begin{array}{l}220 \text { bar } \\
45^{\circ} \mathrm{C} \\
\text { Fração2 }\end{array}$ \\
\hline 1 & 7,767 & alfa-pineno & 4,86 & - & 3,52 & 0,50 \\
\hline 2 & 9,376 & sabineno & 1,28 & - & 1,05 & - \\
\hline 3 & 10,168 & myrceno & 3,88 & - & 3,26 & 0,55 \\
\hline
\end{tabular}




\begin{tabular}{|l|l|l|l|l|l|l|}
\hline $\mathbf{4}$ & 10,820 & alfa-felandreno & 23,3 & 0,19 & 20,47 & 2,58 \\
$\mathbf{5}$ & 11,084 & delta-3-careno & 19,37 & 0,18 & 16,99 & 2,28 \\
$\mathbf{6}$ & 11,651 & para-cimeno & 1,81 & 0,12 & 1,67 & 0,37 \\
$\mathbf{7}$ & 11,935 & limoneno & 21,37 & 0,25 & 19,66 & 2,93 \\
$\mathbf{8}$ & 14,634 & terpinoleno & 1,33 & - & 1,3 & 0,33 \\
$\mathbf{9}$ & 20,028 & Alfa-felandreno-epoxido & 0,32 & 0,87 & 0,38 & 0,87 \\
$\mathbf{1 0}$ & 26,276 & eneldo-pimenta & 0,36 & $4,49-$ & 0,75 & 1,79 \\
$\mathbf{1 1}$ & 27,048 & delta-elemeno & 0,62 & 1,93 & 0,75 & 1,77 \\
$\mathbf{1 2}$ & 28,677 & cariofileno & 1,06 & 4,81 & 1,05 & 3,39 \\
$\mathbf{1 3}$ & 29,825 & germacrene-D & 3,21 & 4,45 & 3,77 & 6,54 \\
$\mathbf{1 4}$ & 32,417 & beta-elemen-7-alfa-ol & 5,91 & 14,37 & 7,44 & 11,68 \\
$\mathbf{1 5}$ & 35,154 & elemol & 4,68 & 42,35 & 8,11 & 15,06 \\
$\mathbf{1 6}$ & 40,004 & beta-elemeno & 6,01 & 23,65 & 8,22 & 17,37 \\
\hline
\end{tabular}

Devido as diferenças quantitativas (\% área no cromatograma), na composição química das frações 1 e 2 nas duas condições de extração analisadas, $\left(80\right.$ bar e $35^{\circ} \mathrm{C} ; 220$ bar e $\left.45^{\circ} \mathrm{C}\right)$ pode-se observar que houve um fracionamento com $\mathrm{CO}_{2}$ supercrítico dos extratos de $S$. terebinthifolius $\mathrm{R}$. nas condições experimentais testadas. Os resultados mostram que, na condição de 80 bar e $35^{\circ} \mathrm{C}$ houve uma melhor separação dos compostos. Isto pode ser observado de maneira qualitativa nos cromatogramas (figura 3 e Figura 4) A fração 1 apresentou uma porcentagem de área no cromatograma alta de monoterpenos (1-8) e baixa em sesquiterpenos (12-16). Já, o cromatograma da fração 2 na mesma condição, observou-se que não houve presença dos primeiros compostos, resultando em uma fração com 7 compostos identificados. Estes resultados sugerem que os compostos com menor peso molecular foram os primeiros a sair deixando por último os mais pesados.
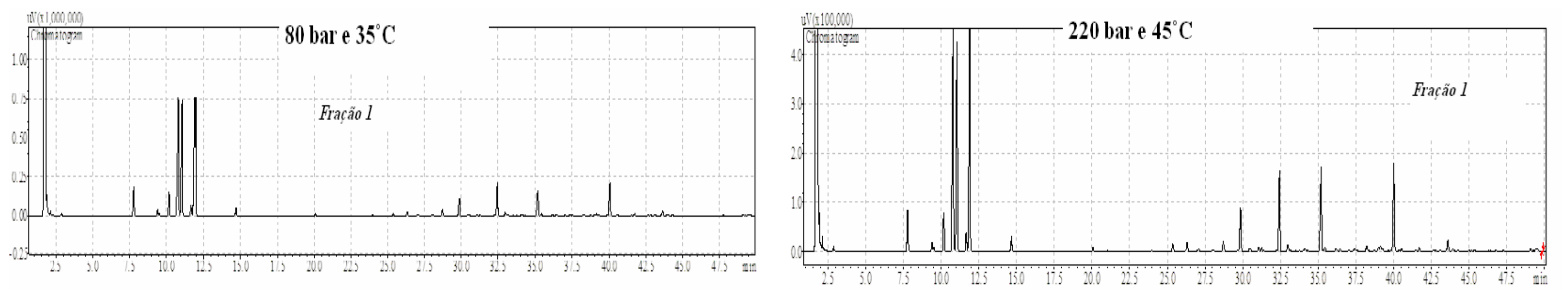

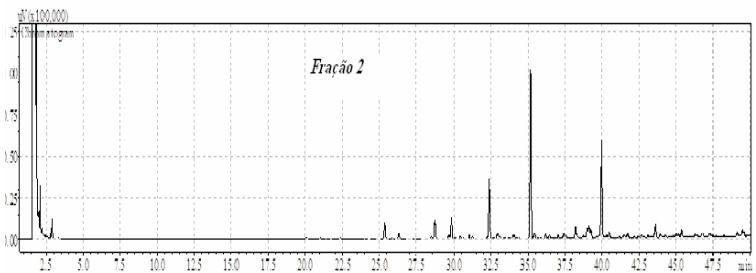

Figura 3 - Cromatograma 220 bar e 45C

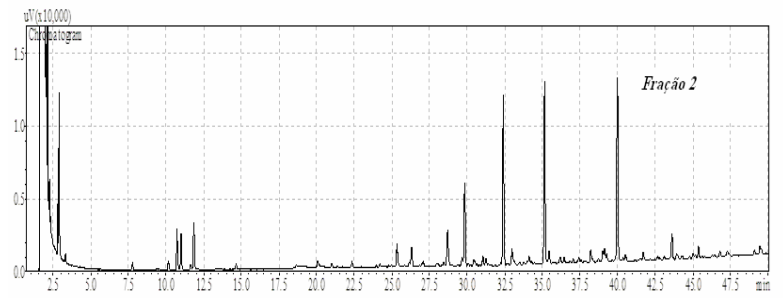

Figura 4 - Cromatograma 220 bar e 45C

Este resultado está de acordo com os estudos realizados por Gupta, et al., (2007), demonstraram que a solubilidade de monoterpenos hidrocarbonados como o limoneno em $\mathrm{CO}_{2}$ supercrítico é maior 
quando se trabalha em condições operacionais menores que 96 bar e $50^{\circ} \mathrm{C}$.

Na condição de 220 bar e $45^{\circ} \mathrm{C}$, observa-se que para fração 1, os primeiros oito compostos identificados se apresentaram em menor proporção em comparação com a fração 2 para condição operacional de. Analisando a fração $2\left(220\right.$ bar e $\left.45^{\circ} \mathrm{C}\right)$, observa-se que os compostos identificados se encontraram em uma maior proporção do que não fração 2 na condição de 80 bar e $35{ }^{\circ} \mathrm{C}$. Este fato pode ser explicado pela perda de seletividade do $\mathrm{CO}_{2}$ supercrítico por se encontrar a uma pressão elevada (BRUNNER, 1994; MUKHOPADHYAY, 2000).

\section{CONCLUSÕES}

Ao utilizar o processo de extração e separação com dióxido de carbono em estado supercrítico, determinou-se que para a obtenção de frações com composição química diferente, a densidade do solvente deve ser mantida em valores baixos com pressões e temperaturas moderadas, a fim de garantir um bom fracionamento dos extratos. Assim, a condição de 80 bar com $35^{\circ} \mathrm{C}$, foi a que apresentou frações mais definidas em sua composição química;

Foram identificados como compostos majoritários na matriz vegetal. Na fração 1: alfa felandrene, delta-3 carene e limonene. Na fração 2: beta-Elemen-7 alfa-ol e elemol;

\section{REFERÊNCIAS}

BRUNNER, G., Gas extraction: an introduction to fundamentals of supercritical fluids and the application to separation processes. Steinkop $\square$, 1994. (Topics in physical chemistry). ISBN 9783798509443.

GAÑÁN, N.; BRIGNOLE, E. A. Supercritical carbon dioxide fractionation of T. minuta and S. officinalis essential oils: Experiments and process analysis. The Journal of Supercritical Fluids, v. 78, p. 12-20, jun. 2013.

GUPTA R.B. and Shim J.J.Solubility in supercritical carbon dioxide. New york : Taylor and Francis Group, 2007. - 1 st..

LORENZI H. Árvores Brasileiras: manual de identificação e cultivo de plantas. São Paulo : Inst. Plantarum de estudos da flora Ltda, 1998. - 8586714070

MUKHOPADHYAY M.Natural extracts using supercritical carbon dioxide. - Washington : CRC Press, 2000.

OCA E. [et al.]Supercritical fluid extraction of different processsed of rosemary plant.J. Agric. Food Chem.. - 1999. - Vol. 47. - p. 1400

SAMUELSSON GunnarDrugs of Natural Origin: A Textbook of Pharmacognosy - Stockholm : [s.n.], 1999.

REVERCHON E., DE MARCO Supercritical fluid extraction and fractionation of natural matter. Journal of Supercritical Fluids. - 2006. - 38. - pp. 146-166. 